\title{
Application of Active Thermography for the Characterization of Building Structures in Cultural Heritage
}

\author{
Christiane Maierhofer ${ }^{1}$, Philipp Myrach ${ }^{1}$ and Rainer Krankenhagen ${ }^{1}$ \\ ${ }^{1}$ BAM Federal Institute for Materials Research and Testing, D-12200 Berlin, Germany \\ Christiane.Maierhofer@bam.de
}

Active thermography has been proven to be very suitable for the investigation of safety relevant structures in civil engineering. It is suitable for the detection of delaminations of any facade elements like plaster, tiles, and natural stone as well as for detecting cracks. The method can also be applied for testing the integrity of any subsequently fitted reinforcement like composite laminates. For detecting inhomogeneities by temperature differences, a nonstationary heat transfer inside the structure has to be initiated. Thus, if the structures to be tested are located outside, also the sun or the daily change of air temperature can be used for testing.

As active thermography using heating with artificial or natural radiation sources is completely non-touching, non-destructive and provides direct images of the investigated area, it is particularly suitable for investigating cultural heritage objects. In this contribution, basic research and case studies will be presented concerning the detection and quantification of delaminations, cracks and further subsurface inhomogeneities in historic facades and historic concrete. Different heating sources like infrared radiators, halogen lamps, fan heater and natural sun radiation are compared.

Keywords: civil engineering, cultural heritage, pulse heating, delamination, crack, sun radiation 